\title{
Critical evaluation of the DNA-methylation markers ABCG1 and SREBF1 for Type 2 diabetes stratification
}

\author{
Christin Krause ${ }^{1}$, Helen Sievert ${ }^{1}$, Cathleen Geißler ${ }^{1}$, Martina Grohs ${ }^{1}$, Alexander T El \\ Gammal $^{2}$, Stefan Wolter ${ }^{2}$, Olena Ohlei ${ }^{3}$, Fabian Kilpert ${ }^{3}$, Ulrike M Krämer ${ }^{5,6}$, Meike \\ Kasten $^{4,7}$, Christine Klein ${ }^{4}$, Georg E Brabant ${ }^{1}$, Oliver Mann ${ }^{2}$, Hendrik Lehnert ${ }^{1,8}$ \& \\ Henriette Kirchner*,1,8 \\ ${ }^{1}$ Medical Department I, Division Epigenetics \& Metabolism, University of Lübeck, Lübeck, Germany \\ ${ }^{2}$ Department of General, Visceral \& Thoracic Surgery, University Medical Center Hamburg-Eppendorf, Hamburg, Germany \\ ${ }^{3}$ Lübeck Interdisciplinary Platform for Genome Analytics, Institutes of Neurogenetics \& Integrative \& Experimental Genomics, \\ University of Lübeck, Lübeck, Germany \\ ${ }^{4}$ Institute of Neurogenetics, University of Lübeck, Lübeck, Germany \\ ${ }^{5}$ Department of Neurology, University of Lübeck, Lübeck, Germany \\ ${ }^{6}$ Institute of Psychology II, University of Lübeck, Lübeck, Germany \\ ${ }^{7}$ Department of Psychiatry \& Psychotherapy, University of Lübeck, Lübeck, Germany \\ ${ }^{8}$ German Center for Diabetes Research (DZD), München-Neuherberg, Germany \\ *Author for correspondence: Tel.: +49 4513101 7847; Fax: +49 04513101 78445; henriette.kirchner@uksh.de
}

Aim: Validation of epigenome-wide association studies is sparse. Therefore, we evaluated the methylation markers cg06500161 (ABCG1) and cg11024682 (SREBF1) as classifiers for diabetes stratification. Patients \& methods: DNA methylation was measured in blood $(n=167)$, liver $(n=99)$ and visceral adipose tissue $(\mathrm{n}=99)$ of nondiabetic or Type 2 diabetic subjects by bisulfite pyrosequencing. Results: DNA methylation at cg11024682 in blood and liver correlated with BMI. Methylation at cg06500161 was influenced by the adjacent SNP rs9982016. Insulin-resistant and sensitive subjects could be stratified by DNA methylation status in blood or visceral adipose tissue. Conclusion: DNA methylation at both loci in blood presents a promising approach for risk group stratification and could be valuable for personalized Type 2 diabetes risk prediction in the future.

First draft submitted: 27 September 2018; Accepted for publication: 11 March 2019; Published online: 6 June 2019

Keywords: ABCG1 • blood • DNA methylation • EWAS • liver • SREBF1 • Type 2 diabetes • visceral adipose tissue

\section{Background}

The increasing incidence of Type 2 diabetes represents one of the most endangering modern epidemics, challenging economics and health systems simultaneously $[1,2]$. Type 2 diabetes is a progression of increasing insulin resistance in combination with failure of pancreatic $\beta$ cells to sufficiently produce insulin. Risk factors for Type 2 diabetes include age, obesity, genetic predisposition, malnutrition and lack of exercise [3]. During disease development abnormal glucose and fatty acid metabolism often manifests before Type 2 diabetes establishes. Moreover, clinical presentation of subjects with Type 2 diabetes or at risk of developing Type 2 diabetes is extremely diverse making it difficult to correctly diagnose and treat those patients [4]. Thus, establishing a quick and robust predictive risk scoring based on novel biomarkers in addition to classical risk factors could lead to personalized intervention strategies before disease manifestation, thereby improving disease outcome and patient care [5].

First genome-wide approaches to calculate a genetic risk score for Type 2 diabetes were based on SNPs significantly associated with blood glucose, insulin resistance itself or BMI, which were identified by genome-wide association studies [4-10]. However, these SNPs explain only a marginal percentage of disease occurrence or heritability [7]. Other attempts to understand the disease etiology of Type 2 diabetes include the study of environmental influences on aberrant gene expression that are mediated by epigenetic alterations, which can be passed throughout generations [1113]. DNA methylation, which generally occurs on cytosines of $\mathrm{CpG}$ oligonucleotides, represents a tissue-specific

Future Medicine 
epigenetic mark, which is often altered in obesity and Type 2 diabetes. The importance of epigenetic modifications in diabetes pathogenesis is highlighted by recent case-control studies that show significant alterations in DNA methylation between nondiabetic and Type 2 diabetic subjects in many tissues of direct relevance for glucose homeostasis on single-loci and genome-wide level [14-18]. Moreover, genome-wide changes in DNA methylation are compared in case-control-based epigenome-wide association studies (EWAS) [19-25] on metabolic diseases. One advantage of EWAS is that their results can be used to generate a methylation risk score (MRS) in order to model additive effects in obesity or diabetes development. For instance, the MRS can be combined with SNP data to improve risk scoring or to access effects of SNPs on DNA methylation [26,27]. Thus, large-scale EWAS could pave the way for the development of robust blood-based biomarker for quick clinical stratification of patients. Table 1 summarizes the lead candidate DNA methylation loci, which were recently identified by large-scale EWAS comparing various ethnicities and clinical characteristics, and which could be exploited for biomarker development and the molecular understanding of Type 2 diabetes etiology. Out of these studies, only two candidate methylation sites for estimating the risk of developing Type 2 diabetes are consistently detected. These are ABCG1 locus cg06500161 and SREBF1 locus cg11024682 [22,23,25-30]. DNA methylation in peripheral blood at these two loci is reported to correlate with BMI and future risk to develop Type 2 diabetes. Because the reported changes in DNA methylation are relatively small and the presented studies are of exceptional high sample size and thus high statistical power, we wanted to test if the two candidate biomarkers, cg06500161 and cg11024682, can be applied for diabetes stratification in smaller cohorts with a wide range of metabolic traits. Therefore, we aimed at evaluating the usefulness of the two methylation markers, ABCG1 locus cg06500161 and SREBF1 locus cg11024682, for the prediction and diagnosis of Type 2 diabetes in the clinical practice and asked the question: can these markers be used to predict or diagnose the incidence of Type 2 diabetes in an individual person? Additionally we wanted to test if DNA methylation of both markers is also altered in tissues regulating glucose homeostasis as DNA methylation patterns and their changes are tissue specific and it is mandatory to carefully analyze candidate methylation loci from blood-based EWAS in metabolically relevant tissues [31].

We measured methylation in DNA extracted from whole blood of a small north German cohort of healthy subjects $(n=176)$ without Type 2 diabetes. For an extensive risk evaluation, we additionally studied SNPs $\pm 200 \mathrm{~kb}$ around each locus in this population. Furthermore, we evaluated the physiological significance of the methylation markers in the metabolically active tissues liver and visceral adipose tissue (VAT) from obese non-diabetic and obese subjects with Type 2 diabetes of another north German cohort $(n=100)$. We used bisulfite pyrosequencing for DNA methylation analysis, the current gold standard, to omit probable bias caused by technical challenges of genome-wide methylation assays [40].

\section{Research design \& methods}

Design \& subjects of metabolic cohort

Liver and VAT biopsies were obtained during bariatric surgery at University Hospital Eppendorf (UKE, Hamburg, Germany). All subjects were categorized by their HbAlc value into healthy (HbAlc $<48 \mathrm{mmol} / \mathrm{mol}, 6.5 \%$ ) or subjects with overt Type 2 diabetes (HbA1c $\geq 48 \mathrm{mmol} / \mathrm{mol}, 6.5 \%$ ). Clinical characteristics are listed in Supplementary Table 1.

\section{Design \& subjects of blood cohort}

Blood samples were collected during a study to evaluate the relationship between obesity and eating behavior. Subjects of this cohort were healthy overweight or lean men and women from north Germany without diagnosed Type 2 diabetes. The exclusion criteria for the prospective study in which blood samples were collected comprised diabetes, hyper- or hypothyroidism, current major depression or mania, anorexia, bulimia, binge eating disorder, substance abuse more frequent than three-time/month, alcohol abuse (audit $>10$ ) and medications (tricyclic antidepressants, atypical antipsychotics, $\beta$-blocker, corticosteroids, valproate, lithium, amphetamines, laxatives). These criteria were chosen to exclude participants with known factors influencing eating behavior or BMI, such as thyroid disorders or current major depression. Clinical characteristics are listed in Supplementary Table 1.

\section{Clinical parameters}

Blood was drawn after an overnight fast and clinical parameters were measured in by the Institut für Klinische Chemie und Laboratoriumsmedizin, Zentrum für Diagnostik, Universitätsklinikum Eppendorf, Hamburg, Germany according to the DIN EN ISO 15189:2014 certification (tissue cohort). Clinical parameters of the 


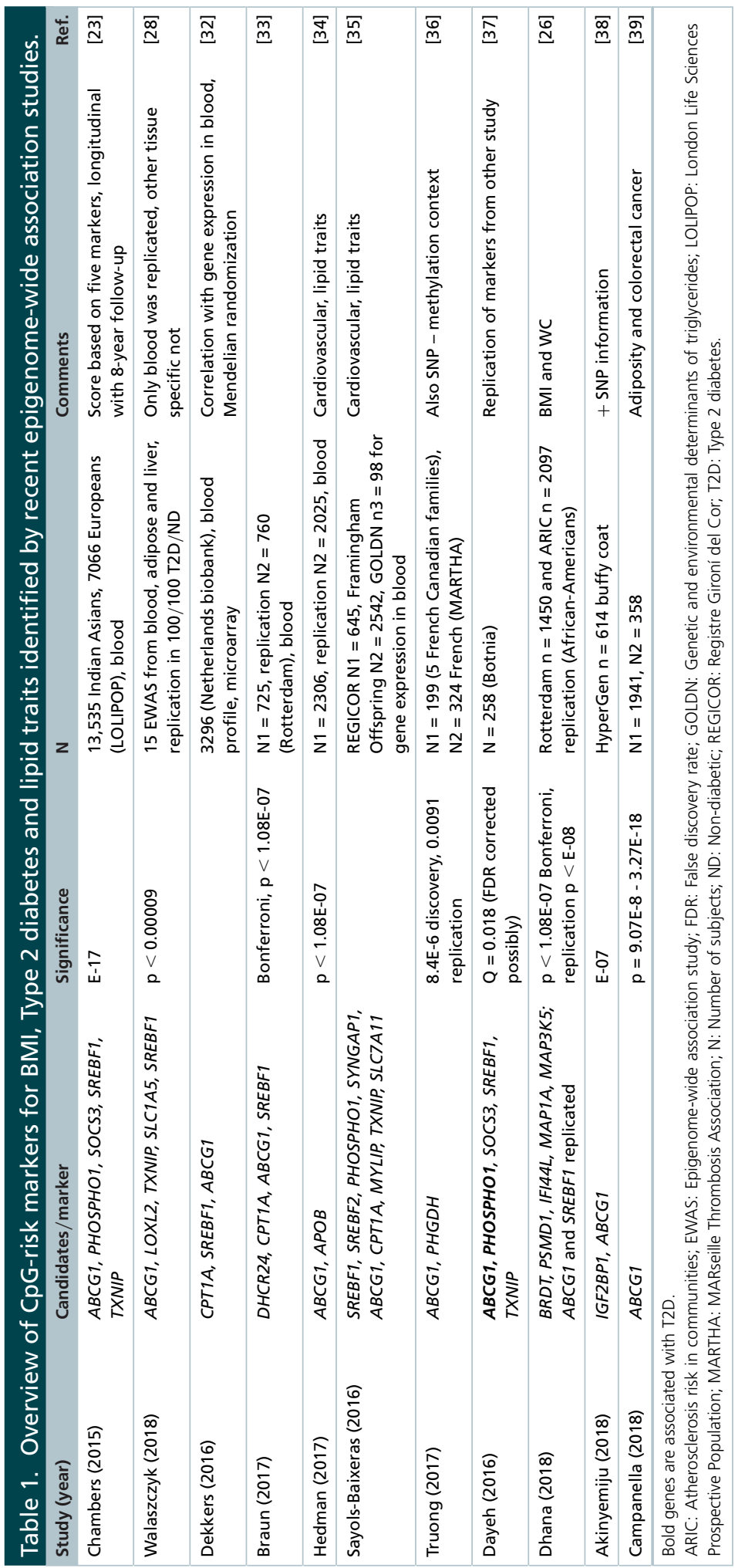


blood cohort were analyzed from frozen serum by the LADR Der Laborverbund Dr Kramer \& Kollegen GbR, 21502 Geesthacht, Germany according to the DIN 9001:2008. Glucose, glutamate oxaloacetate transaminase, glutamic pyruvic transaminase, gamma-glutamyltransferase, creatinine, alkaline phosphatase, cholesterol, highdensity lipoprotein and triglycerides were determined using photometric assays, HbA1c was quantified using capillary electrophoresis or turbidimetric inhibition assays and folic acid and thyroid stimulating hormone were measured using luminescent oxygen channeling immunoassays. Insulin was measured from frozen serum using the Human/Canine/Porcine Insulin Quantikine ELISA kit (R\&D Systems, MN, USA).

\section{DNA isolation}

For the blood cohort, DNA was extracted from whole blood by separation of leucocytes and denaturation of cellular proteins by Proteinase K treatment. DNA was precipitated and purified by addition of saturated sodium chloride solution, isopropanol and ethanol. DNA stock solutions were prepared in TE buffer.

DNA from VAT and liver was extracted by QIAmp DNA mini kit (QIAGEN, Venlo, The Netherlands) as indicated by manufacturer with minor changes. In short, $25 \mathrm{mg}$ of frozen liver or VAT were homogenized in $80 \mu \mathrm{l}$ phosphate buffered saline (Bead Ruptor Omni International) and $180 \mu \mathrm{l}$ tissue lysis buffer was added. The homogenate was incubated with Proteinase $\mathrm{K}$ for $1 \mathrm{~h}$ and stored before DNA extraction for a maximum of 1 month at room temperature.

\section{DNA methylation measurement \& assay validation}

Extracted DNA was bisulfite converted (bisDNA) by EpiTect Bisulfite kit (QIAGEN, Venlo, The Netherlands) as indicated by the manufacturer. Bisulfite PCR was performed with the Pyromark PCR kit (QIAGEN, Hilden, Germany) with 15-20 ng of input bisDNA for 45 cycles. For cg11024682, previously published primers were used after methylation assay validation [27]. For cg06500161, newly designed primers (fwd: GGTTAGGAGTTTAAAAGGTTGAGTA, rev: biotin-ATCCCCAAAACCTAAAACCACCTCAATAA, seq: AAATTATGTTTAAAGGAAT, sequence to analyze: TAGWTTYGTYGGGTGTTT) had to be used due to primer dimers of previously published primers [27]. Primers were designed by PyroMark Assay Design 2.0 software.

Assay validation included measurement of fully, semi- and nonmethylated bisulfite-converted standard DNA for measurement of DNA methylation sensitivity. This fully and nonmethylated standard bisDNA was purchased (EpiTect Control DNA; Qiagen, Hilden, Germany), and semi-methylated standard bisDNA was generated by mixing equal amounts of non-methylated and fully methylated standard bisDNA. For bisulfite PCR, $10 \mathrm{ng}$ of each standard bisDNA sample was used. Further analysis included primer controls for pyrosequencing to exclude a bias caused by background signals of primer dimers or self annealing. These controls consisted of pyrosequencing reactions including only reverse/sequencing primer, reverse primer only, sequencing primer only and PCR product without additional primers.

\section{Genotyping}

DNA of 122 subjects from the blood cohort was genotyped using a gene-set-assessment chip with custom content (Illumina, CA, USA) (Supplementary Table 5). DNA from the metabolic cohort was specifically genotyped at SNP rs 9982016 by pyrosequencing.

\section{Statistics}

Statistical analysis was performed using Prism 7.02 (GraphPad Software Inc., CA, USA) and MATLAB R2018a (The MathWorks, MA, USA). All calculations were performed after clearance of outliers by robust regression and outlier removal (ROUT) function.

\section{DNA methylation analysis}

For DNA methylation analysis of insulin-resistant and -sensitive subjects, a nonparametric Mann-Whitney test was performed and presented as mean \pm standard deviation. Correlations between DNA methylation and BMI were performed using Pearson's correlation. For the effect size $\beta$ of DNA methylation on BMI, blood glucose, Homeostatic Model Assessment for Insulin Resistance (HOMA-IR, blood cohort) or Hb1Ac (metabolic cohort), a linear regression model with and without correction for age and gender was used. The effect size is given as change of unit outcome for $1 \%$ change in DNA methylation. 
Only polymorphisms in accordance to Hardy-Weinberg equilibrium were considered for association testing. Therefore, a $\chi^{2}$ test with $\alpha=0.05$ was used. A linear regression model with stepwise regression and interaction terms between all pairs of predictors (gender, age and genotype of a certain polymorphism) was used for association between polymorphisms and BMI or polymorphisms and DNA methylation. An additive effect of alleles was assumed. Correction for the correlation between metabolic traits and DNA methylation for confounding factors age and gender, coded as 1 or 2 , was performed by linear regression models:

$$
\text { Trait }=\beta 0+\beta 1 * \mathrm{cg}+\beta 2 * \text { age }+\beta 3 * \text { gender }
$$

Correction for the genotype at rs9982016 was also performed by linear regression, coding the genotype as 1 (GG), 2 (GA) or $3(\mathrm{AA})$ :

$$
\text { Trait }=\beta 0+\beta 1 * \mathrm{cg}+\beta 2 * \text { age }+\beta 3 * \text { gender }+\beta 4 * \text { genotype }
$$

Correction for multiple testing was not performed because all measurements were independent from each other (other assay systems and different kind of samples).

\section{Methylation risk score}

A basic MRS was calculated by summing up standardized values (z) of DNA methylation $\mathrm{x}:=\frac{x-\bar{x}}{S D(x)}$; $M R S=z_{c g 06500161}+z_{c g 11024682}$. Student's t-test was performed to analyze the significance of these MRS. Effect sizes of MRS on other outcomes were calculated by linear regression, whereby a change of MRS about 1 score equals a change of DNA methylation at one CpG site about one standard deviation if DNA methylation at the other position stays constant.

\section{Results}

\section{DNA methylation of the candidate markers \& metabolic traits}

Two markers for obesity and Type 2 diabetes risk prediction were identified by blood-based EWAS, which are ABCG1 locus cg06500161 and SREBF1 locus cg11024682 (Table 1). In order to test if these markers are possible biomarkers for Type 2 diabetes stratification we analyzed the DNA methylation in peripheral blood of 176 healthy subjects (blood cohort) and in the metabolically active liver tissues and VAT of another obese cohort ( $\mathrm{n}=100$; metabolic cohort).

First, we analyzed if methylation at cg06500161 and cg11024682 in DNA extracted from blood, liver or VAT correlates with BMI or any clinical parameter of glucose homeostasis such as fasting blood glucose or HbA1c. To do so, we evaluated both bisulfite pyrosequencing assays that were previously used to validate EWAS findings [27]. The SREBF1 assay showed low single peak height but no background signal that is often caused by primer dimer between sequencing and reverse primer. However, a previously published ABCG1 assay exhibited a high primer dimer background (Supplementary Figure 1B) and was therefore not used in our study. Consequently, we used the published SREBF1 assay and a newly designed ABCG1 assay that did not generate background signals. Both pyrosequencing assays appeared to be biased toward unmethylated strands (Supplementary Figure 1A). Sequencing of non-, semi- and fully methylated human control DNA revealed a DNA methylation detection range of 6, 33 and $84 \%$ for SREBF1 and 6, 35 and $86 \%$ for $A B C G 1$, respectively.

In liver tissue, methylation at cg1 1024682 (SREBF1) showed a significant negative correlation with BMI with a rather weak correlation coefficient $(\mathrm{p}=0.0442, \mathrm{r}=-0.2103$; Figure $1 \mathrm{~A})$. To the contrary, the same position correlated weakly positive with BMI in blood-derived DNA from our blood cohort $(\mathrm{p}=0.0211, \mathrm{r}=0.2408$; Figure 1E). For SREBF1 we detected a significant effect of blood DNA methylation on blood glucose levels ( $\mathrm{p}=0.0016$ after correction for age and gender), which could not be reproduced in our liver and VAT cohort (Supplementary Table 2). For cg06500161 (ABCG1) no significant correlation between DNA methylation and BMI was found in any tissue (Figure 1B, D \& F). The only significant correlations for $A B C G 1$ could be found between glucose levels and DNA methylation in blood ( $\mathrm{p}=0.0007$ after correction for age and gender) and HOMA-IR ( $\mathrm{p}=0.0165$ after correction for age and gender).

To analyze whether DNA methylation is affected by disease state independently of BMI, we divided our liver and VAT cohort into healthy obese (HbA1c $<6.5 \%, 48 \mathrm{mmol} / \mathrm{mol}$ ) and obese with Type 2 diabetes (HbA1c $\geq 6.5 \%$, $48 \mathrm{mmol} / \mathrm{mol}$ ). Because our blood cohort consisted only of nondiabetic subjects we had to divide this group into 
(A)

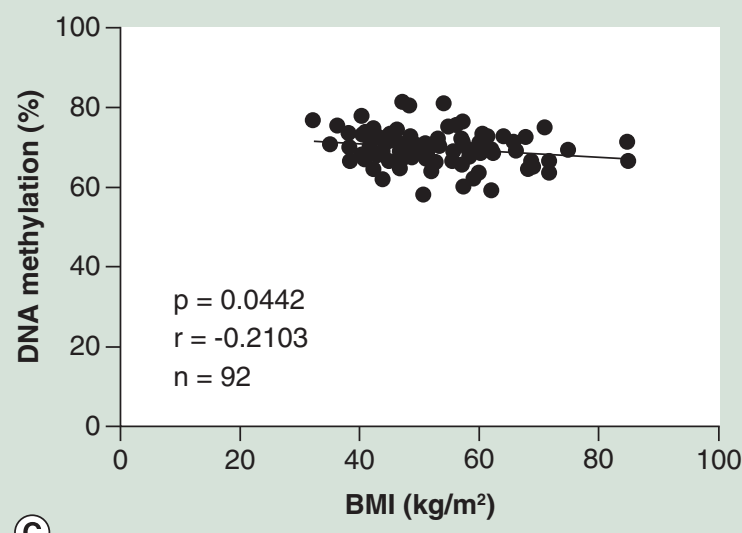

(C)

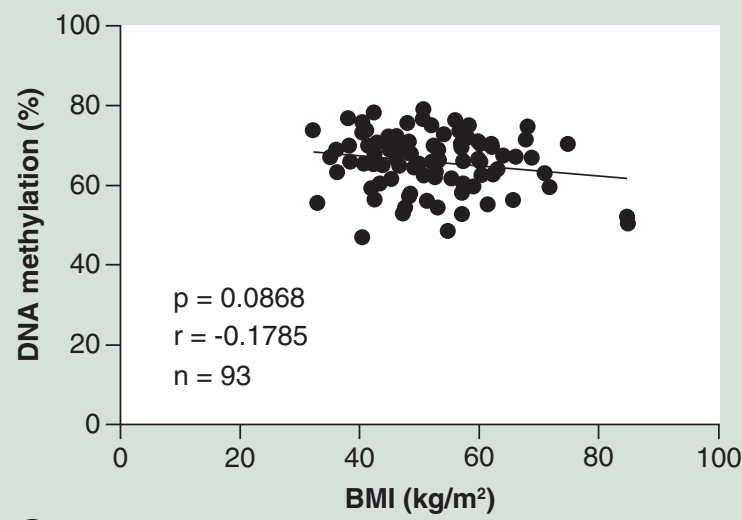

(E)

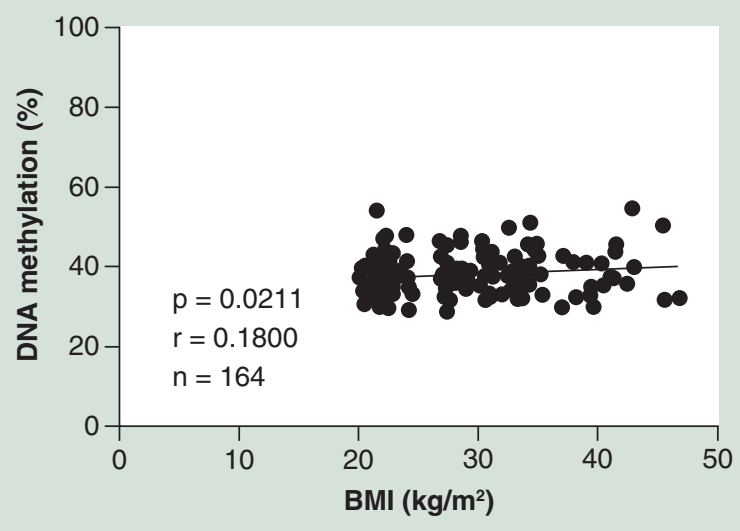

(B)

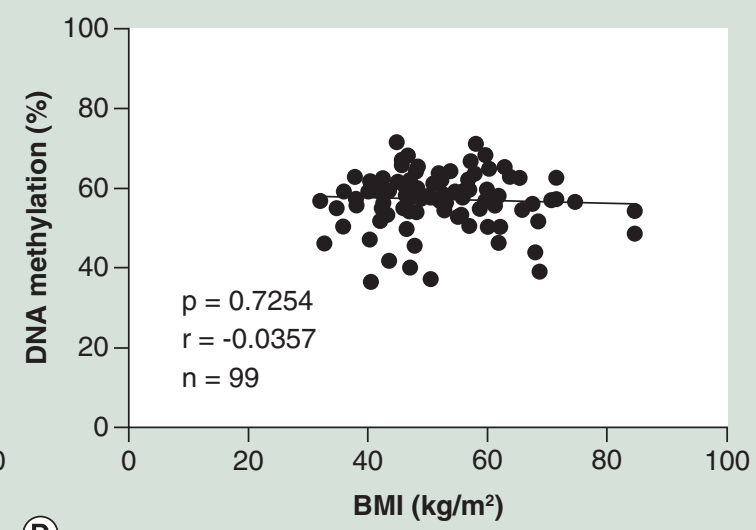

(D)

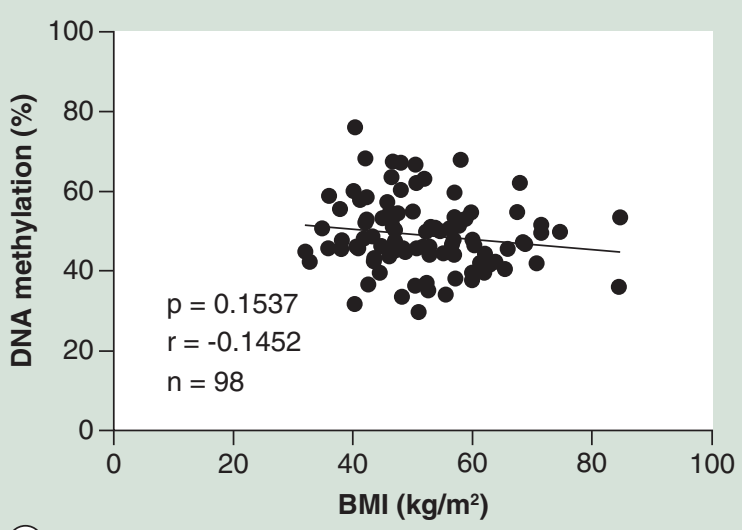

(F)

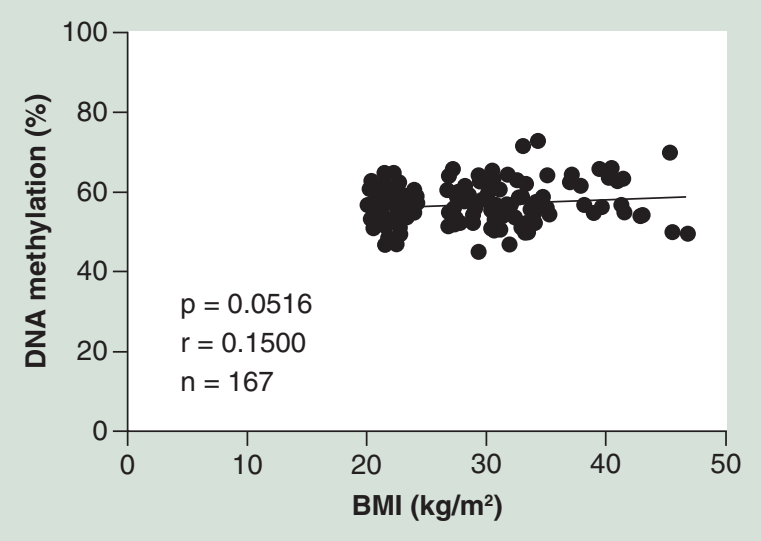

Figure 1. Pearson correlation between BMI and DNA methylation. Methylation at cg11024682 (SREBF1) or cg06500161 (ABCG1) was measured in liver (A \& B), visceral adipose tissue (C \& D) and blood (E \& F). Only SREBF1 methylation in liver (A) and blood (E) correlates significantly with BMI.

insulin sensitive (HOMA-IR $<2.5$ ) and insulin resistance (HOMA-IR $\geq 2.5$ ) without diagnosed Type 2 diabetes (HbA1c $<6.5 \%, 48 \mathrm{mmol} / \mathrm{mol}$ ). Insulin resistant or subjects with Type 2 diabetes had a slightly higher DNA methylation at both positions reaching significance in blood-derived DNA (Figure 2A \& B). Though, the difference in DNA methylation between groups was marginal with $1.58 \%$ for $S R E B F 1$ and $1.78 \%$ for $A B C G 1$.

To investigate a possible influence of the adjacent SNP rs9982016 (A > T, minor allele frequency [MAF] $=6.63 \%$ on 1000 genomes) on DNA methylation at cg06500161, all subjects were genotyped at this position by either pyrosequencing (metabolic cohort) or by a gene-set-assessment chip (Illumina) (blood cohort) and divided 
(A)

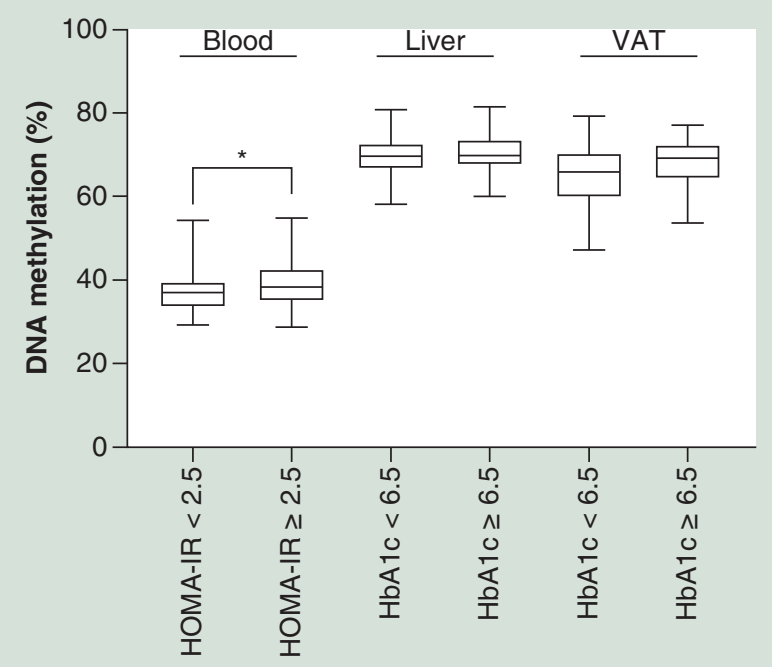

(C)

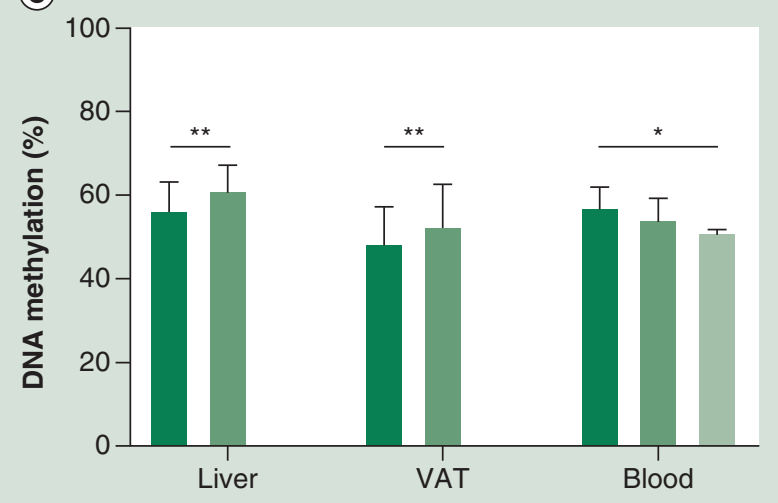

(B)

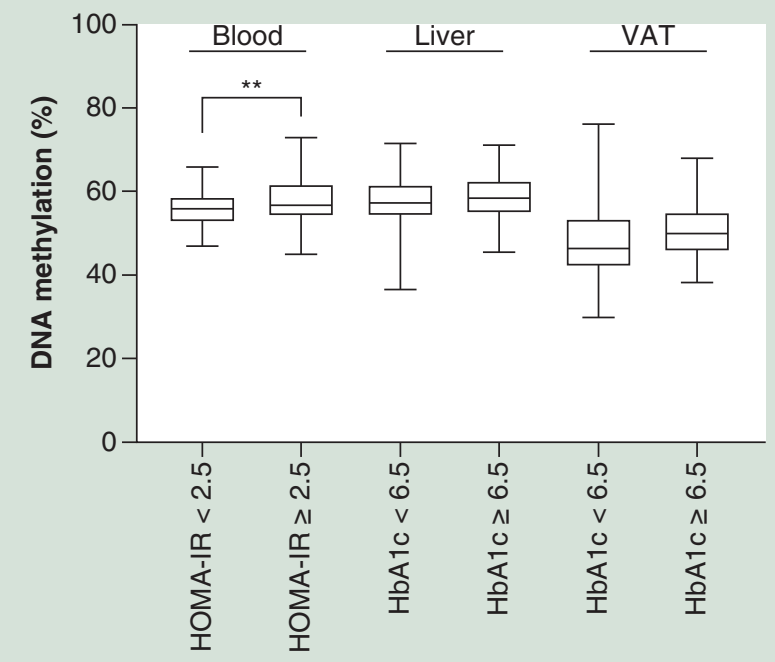

(D)

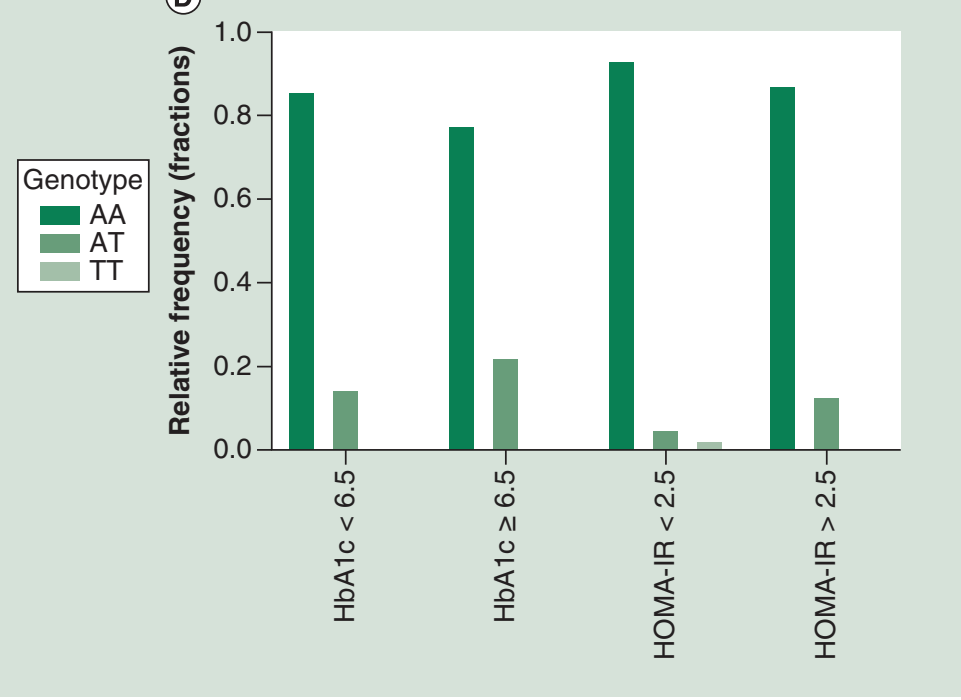

Figure 2. DNA methylation of cg11024682 (SREBF1) and cg06500161 (ABCG1) by pheno- and genotype. Average methylation of cg11024682 (A, SREBF1) and cg06500161 (B, ABCG1) in blood, liver and VAT after categorizing subjects into susceptibility to insulin resistance and diabetic state. Only in blood, DNA methylation differs significantly at cg11024682 (A, $p=0.0194)$ and cg06500161 (B, $p=0.0098$ ). Average methylation of cg06500161 in liver, VAT and blood in dependence of the genotype at rs9982016 (C). For all three tissues, DNA methylation differs significantly within the genotypes AA, AT or TT (liver $p=0.002$, VAT $p=0.0442, b l o o d p=0.0203$ ). Frequency of each genotype of ABCG1 rs9982016 stratified by HbA1c (metabolic cohort) or HOMA-IR (blood cohort) as measurement of insulin resistance (D).

VAT: Visceral adipose tissue.

into their respective genotypes AA, AT and TT. We could detect a significant difference in DNA methylation between the different genotypes of $4.96 \%$ in liver, $7.95 \%$ in VAT and $6.12 \%$ in blood (Figure 2C). Interestingly, the polymorphism appears to affect DNA methylation into different directions but stays consistent with the major A allele being the risk allele for increased BMI by causing a higher methylation in blood or decreased methylation in liver and VAT. In our liver and VAT cohort, we found an MAF of 6.92\% in healthy subjects (AT genotype 13.85\%) and $11.42 \%$ (AT genotype 22.86\%) in subjects with Type 2 diabetes (Figure 2D). No subject was homozygous for the $\mathrm{T}$ allele in this cohort. A $\chi^{2}$ test revealed no allelic association to this polymorphism and the incidence of Type 2 diabetes for the metabolic cohort or association of this polymorphism with obesity for our blood cohort. Addition of rs9982016 as cofactor for linear regression (Supplementary Table 3) resulted into a significant correlation between 
blood DNA methylation at cg06500161 and BMI ( $\mathrm{p}=0.0123$ after correction for age, gender and genotype for rs9982916).

Previously it was shown that DNA methylation of both markers in blood-derived DNA correlates with DNA methylation of other tissues [27]. Therefore, we tested whether DNA methylation within VAT and liver of our metabolic cohort is uniform. We did not observe this relationship (data not shown), indicating a highly tissuespecific DNA methylation pattern for both sites.

\section{Generation \& analysis of a methylation risk score based on two methylation markers}

Since Type 2 diabetes is a multifactorial disorder, we were interested whether a combination of both measured methylation markers would be a useful biomarker in the context of risk group stratification. Therefore, we calculated a basic MRS by standardized methylation values of ABCG1 locus cg06500161 and SREBF1 locus cg11024682. Furthermore, we evaluated the distribution of this score in our cohorts divided into obese and lean (by BMI) subjects, insulin-resistant or -sensitive (by HOMA-IR) subjects or diagnosis of Type 2 diabetes. For the blood cohort, we included 159 subjects for association of MRS with BMI and 127 subjects for association of MRS with HOMA-IR. For the metabolically active tissues, we included 92 subjects from the liver and 93 subjects from the VAT cohort stratified by HbA1c values into obese subjects with or without Type 2 diabetes (Supplementary Table 4).

In the blood cohort, stratification of the subjects either into obese and lean, or into insulin-sensitive and -resistant subjects resulted in a significant difference in means of MRS ( $\mathrm{p}=0.0292$ for BMI; and $\mathrm{p}=0.0015$ for HOMA-IR; Supplementary Table 4). Due to the strong overlap of both distributions, it was not possible to define certain phenotyping quantiles. However, the basic MRS showed significant correlations and effect sizes for blood glucose, HOMA-IR and BMI ( $\mathrm{p}=0.0162, \beta=0.7790$ for BMI; $\mathrm{p}=1.04 \mathrm{E}-04, \beta=0.3359$ for HOMA-IR and $\mathrm{p}=1.28 \mathrm{E}-06$, $\beta=2.2530$ for blood glucose). Next, we generated a receiver operating characteristic (ROC) curve and analyzed the area under the curve (AUC) to evaluate the detection accuracy of our MRS. Stratification of subjects from the blood cohort by BMI based on the blood-methylation derived MRS resulted in an AUC of 0.5938. Stratification of the same subjects by HOMA-IR resulted in an AUC of 0.676.

In the metabolic cohort, calculating an MRS showed a significant difference in the mean scores of both groups in VAT ( $\mathrm{p}=0.0193$ ), but not in liver ( $\mathrm{p}=0.0709$, Supplementary Table 4). Unfortunately, it was not possible to calculate certain score quintiles due to the same distribution of the scores. Nevertheless, the MRS displayed a significant correlation with and effects on BMI $(p=0.0335, \beta=-1.5417$ for VAT and $p=0.0438, \beta=-1.4391$ for liver). The AUC of the ROC curve for stratification of subjects from the metabolic cohort by HbA1c based on the VAT methylation-derived MRS was 0.6419. Stratification by HbA1c based on the liver methylation-derived MRS yielded an AUC 0.5880. Our results show that a statistical combination of marginal changes in DNA methylation $(1-2 \%)$ is able to mirror the context between DNA methylation and obesity or insulin resistance throughout tissues, even though we analyzed only two candidate CpG sites.

\section{Discussion}

In the recent years it has become more and more evident that in addition to genetic factors epigenetic modifications, either inherited or acutely acquired, contribute to the development of metabolic diseases including obesity and Type 2 diabetes [41,42]. EWAS are therefore important milestones, moving the entire field ahead. To test if EWAS-derived markers are useful for individual patients in terms of biomarker development for diabetes prediction and diagnosis we validated two recent EWAS markers. The EWAS top hits analyzed here, ABCG1 and SREBF1, have putative metabolic functions and likely contribute to the etiology of Type 2 diabetes on a molecular level. $A B C G 1$ which is highly expressed in adipose tissue and promotes the uptake of triglyceride into adipocytes. In liver and macrophages it might play a regulating role of tissue lipid levels [43]. Obesity is associated with overexpression of $A B C G 1$, at the same time complete loss of function in mice is protective against diet-induced obesity [44,45]. SREBP1c is a major insulin-induced transcription factor in liver and VAT regulating lipogenesis by binding regulatory elements of the $L D L R$ or of other genes contributing to cholesterol synthesis and uptake [46]. Previous studies in adipose tissue of obese subjects showed a significant downregulation of gene expression, which can be reversed after weight loss [47].

Blood DNA methylation at both analyzed CpG sites ABCG1 locus cg06500161 and SREBF1 locus cg11024682 correlates significantly with blood glucose level as previously reported, even in our two cohorts consisting of patients with various metabolic traits. After stratification of our blood cohort into insulin-sensitive (HOMA-IR $<2.5)$ and 
-resistant (HOMA-IR $\geq 2.5$ ) subjects, the latter group revealed an increased DNA methylation at both positions, in accordance to methylation in liver and VAT for subjects with Type 2 diabetes. However, DNA methylation in blood and both metabolic tissues correlates inversely with BMI leading to the conclusion that blood is not a suitable surrogate tissue for mirroring obesity-induced metabolic changes leading to or caused by aberrant DNA methylation. This conclusion is in agreement with another study previously published [23,28]. Large-scale EWAS are therefore likely not appropriate to identify the molecular mechanisms of disease pathogenesis. Often minor changes in DNA methylation, which are probable not of physiological relevance, become statistically significant only driven by the large sample sizes of EWAS. Thus, EWAS data are not always 'translatable' to smaller groups and individuals because stratification of subjects becomes difficult when the difference between groups is only marginal. Thus, reliable clinical biomarker for individual patients can currently not be deducted from EWAS. Nevertheless, EWAS might work in the future for identification of disease biomarker in a surrogate tissue, as for biomarker it is not a necessity that the tissue or marker itself is physiologically involved in disease etiology as long as the marker is robust and reliable. For the future, biomarker development should be focused, however, on markers that show greater differences in DNA methylation between groups.

It should be noted that we were not able to obtain blood DNA from the metabolic cohort in which we studied DNA methylation in liver and VAT. While our blood cohort consisted of healthy nondiabetic subjects with a wide range of BMIs, the liver and VAT biopsies were obtained during weight-loss surgery of a severely obese and metabolic diseased cohort. Thus, caution should be applied when comparing methylation of blood DNA with results derived in liver and VAT. A further limitation of our study is that we did not account for differences in DNA methylation due to changes in cell-type composition [48,49]. Because the two methylation markers investigated here were originally discovered in EWAS that are based on DNA from whole blood or buffy coat (Table 1) we did not perform cell sorting to be comparable with these data. Adjustment for cell types based on epigenetic profiles cannot be performed respectively [50] in our study as we did not measure DNA methylation on a genome-wide level. However, we could not find correlations of DNA methylation with white blood cell count or C-reactive protein, which are marker for inflammation (Supplementary Table 6). Therefore, we estimate that here presented results are not solely due to changes in cell-type composition.

The SNP rs9982016 located in proximity to cg06500161 within intron 4 of $A B C G 1$ is contradictorily reported to either correlate with BMI by influencing DNA methylation or to not correlate with BMI, dependent of the analyzed population $[22,25,27]$. Our results indicate an influence of this polymorphism on DNA methylation independently of tissue type. The SNP itself is not associated to BMI but after inclusion as cofactor for linear regression we observe a significant association between blood DNA methylation and BMI. Weighted genetic risk scores based on SNP information are useful tools for disease stratification [51-53]. On the other hand, generation of an MRS can be of advantage as it leads to one highly significant variable for univariate and bivariate analysis and was successfully tested for cancer [54].

\section{Conclusion}

The calculation of a basic MRS based on DNA methylation at cg06500161 and cg11024682 is able to stratify our blood cohort significantly into insulin-resistant and -sensitive or lean and obese subjects. This result is supported by generation of an ROC curve. Nevertheless, the predictive and/or diagnostic value of measuring these methylation markers is too small to justify them as a novel biomarker for the use in individuals. Thus, further improvement should be achieved by including CpG sites that show a higher difference in methylation and weighting of each contribution to the score by properly examined effect sizes.

\section{Future perspective}

Even though aberrant methylation at $\operatorname{cg} 06500161$ and $\operatorname{cg} 11024682$ in blood might not mirror methylation changes in metabolically active tissues and is likely not involved in diabetes pathology on a molecular level, our data reproduced an association of blood DNA methylation and increased blood glucose and development of insulin resistance beyond large EWAS. The observed differences in DNA methylation at both markers in blood are too small to use those as a robust novel biomarker for diabetes stratification alone. However, combining the blood methylation information with the genotype at risk alleles and other clinical traits adds up to an improved scoring system that is worth to be exploited in the future. For future biomarker development it should be recommended nevertheless to focus on EWAS marker that demonstrates larger methylation differences between groups and that can be validate in small cohorts and ultimately individuals. 


\section{Summary points}

- Two epigenome-wide association studies (EWAS) candidate methylation markers cg06500161 (ABCG1) and cg11024682 (SREBF1) are tested for diabetes stratification in two small cohorts.

- DNA methylation of both markers in liver and visceral adipose tissue does not correlate with metabolic traits.

- Methylation at cg11024682 in blood is associated with BMI.

- EWAS candidate markers are likely not involved in diabetes pathogenesis but provide a starting point for biomarker development.

- Measurement of EWAS candidate marker alone is not sufficient for Type 2 diabetes stratification in small cohorts and individuals.

- EWAS candidate markers with larger differences in DNA methylation between groups need to be prioritized.

- A methylation-risk score based on epigenetics can stratify insulin-sensitive and -resistant subjects.

Supplementary data

To view the supplementary data that accompany this paper please visit the journal website at: www.futuremedicine.com/doi/sup pl/10.2217/epi-2018-0159

Availability of data \& materials

The datasets used and/or analyzed during the current study are available from the corresponding author on reasonable request.

Authors' contributions

$\mathrm{C}$ Krause and $\mathrm{H}$ Kirchner designed experiments and assays, collected and analyzed data, and wrote the paper. H Sievert designed experiments and assays, collected and analyzed data. M Grohs, C Geißler, O Ohlei and F Kilpert collected and analyzed data. AT El Gammal, S Wolter, UM Krämer, M Kasten, C Klein, GE Brabant, O Mann and H Lehnert collected tissues and data. All authors read and approved the final manuscript.

\section{Acknowledgements}

The authors would like to acknowledge CM Lill and L Bertram from Lübeck Interdisciplinary Platform for Genome Analytics, Institutes of Neurogenetics and Integrative and Experimental Genomics for providing gene-set-assessment chip information.

\section{Financial \& competing interests disclosure}

This work was supported by funding from the Deutsche Forschungsgemeinschaft (KI 1887/2-1 and T-CRC-134). The authors have no other relevant affiliations or financial involvement with any organization or entity with a financial interest in or financial conflict with the subject matter or materials discussed in the manuscript apart from those disclosed.

No writing assistance was utilized in the production of this manuscript.

\section{Ethical disclosure}

The authors state that they have obtained appropriate institutional review board approval and have followed the principles outlined in the Declaration of Helsinki for all human experimental investigations. In addition, for investigations involving human subjects, informed consent has been obtained from the participants involved. The study was approved by the local ethics committee of the University Hospital Schleswig-Holstein Campus Lübeck (AZ 13-159) and of the University Hospital Hamburg Eppendorf (PV4889).

\section{Open access}

This work is licensed under the Attribution-NonCommercial-NoDerivatives 4.0 Unported License. To view a copy of this license, visit http://creativecommons.org/licenses/by-nc-nd/4.0/

\section{References}

Papers of special note have been highlighted as: $\bullet$ of interest; $\bullet \bullet$ of considerable interest

1. Jacobs E, Hoyer A, Brinks R, Icks A, Kuß O, Rathmann W. Healthcare costs of Type 2 diabetes in Germany. Diabet. Med. 34(6), 855-861 (2017).

2. Hussain A, Claussen B, Ramachandran A, Williams R. Prevention of Type 2 diabetes: a review. Diabetes Res. Clin. Pract. 76(3), 317-326 (2007).

3. Stumvoll M, Goldstein BJ, van Haeften TW. Type 2 diabetes: pathogenesis and treatment. Lancet 371(9631), 2153-2156 (2008). 
4. Ahlqvist E, Storm P, Käräjämäki A et al. Novel subgroups of adult-onset diabetes and their association with outcomes: a data-driven cluster analysis of six variables. Lancet Diabetes Endocrinol. (2018). http://www.thelancet.com/journals/landia/article/PIIS2213-8587(18)30051-2/abstract

- Highlights the diversity of subjects with Type 2 diabetes and the need for personalized therapies.

5. Meigs JB, Shrader P, Sullivan LM et al. Genotype score in addition to common risk factors for prediction of Type 2 diabetes. N. Engl. J. Med. 359(21), 2208-2219 (2008).

6. van Hoek M, Dehghan A, Witteman JCM et al. Predicting Type 2 diabetes based on polymorphisms from genome-wide association studies: a population-based study. Diabetes 57(11), 3122-3128 (2008).

7. Speliotes EK, Willer CJ, Berndt SI et al. Association analyses of 249,796 individuals reveal 18 new loci associated with body mass index. Nat. Genet. 42(11), 937-948 (2010).

8. Andersson EA, Allin $\mathrm{KH}$, Sandholt $\mathrm{CH}$ et al. Genetic risk score of 46 Type 2 diabetes risk variants associates with changes in plasma glucose and estimates of pancreatic-cell function over 5 years of follow-up. Diabetes 62(10), 3610-3617 (2013).

9. Cornelis MC, Qi L, Zhang C et al. Joint effects of common genetic variants on the risk for Type 2 diabetes in US men and women of European ancestry. Ann. Intern. Med. 150(8), 541-550 (2009).

10. Lango H, The UK Type 2 Diabetes Genetics Consortium, Palmer CN et al. Assessing the combined impact of 18 common genetic variants of modest effect sizes on Type 2 diabetes risk. Diabetes 57(11), 3129-3135 (2008).

11. Schadt EE. Molecular networks as sensors and drivers of common human diseases. Nature 461(7261), 218-223 (2009).

12. Tomar AS, Tallapragada DSP, Nongmaithem SS, Shrestha S, Yajnik CS, Chandak GR. Intrauterine programming of diabetes and adiposity. Curr. Obes. Rep. 4(4), 418-428 (2015).

13. Nilsson E, Ling C. DNA methylation links genetics, fetal environment, and an unhealthy lifestyle to the development of Type 2 diabetes. Clin. Epigenetics 9(1) (2017). http://clinicalepigeneticsjournal.biomedcentral.com/articles/10.1186/s13148-017-0399-2

14. Volkmar M, Dedeurwaerder S, Cunha DA et al. DNA methylation profiling identifies epigenetic dysregulation in pancreatic islets from Type 2 diabetic patients: DNA methylation profiling of Type 2 diabetic islets. EMBO J. 31(6), 1405-1426 (2012).

15. Kirchner H, Sinha I, Gao H et al. Altered DNA methylation of glycolytic and lipogenic genes in liver from obese and Type 2 diabetic patients. Mol. Metab. 5(3), 171-183 (2016).

16. Volkov $\mathrm{P}, \mathrm{Bacos} \mathrm{K}$, Ofori JK et al. Whole-genome bisulfite sequencing of human pancreatic islets reveals novel differentially methylated regions in Type 2 diabetes pathogenesis. Diabetes 66(4), 1074-1085 (2017).

17. Barres R, Kirchner H, Rasmussen $\mathrm{M}$ et al. Weight loss after gastric bypass surgery in human obesity remodels promoter methylation. Cell Rep. 3(4), 1020-1027 (2013).

18. Nilsson E, Matte A, Perfilyev A et al. Epigenetic alterations in human liver from subjects with Type 2 diabetes in parallel with reduced folate levels. J. Clin. Endocrinol. Metab. 100(11), E1491-E1501 (2015).

19. Rakyan VK, Down TA, Balding DJ, Beck S. Epigenome-wide association studies for common human diseases. Nat. Rev. Genet. 12(8), 529-541 (2011).

20. Xu X, Su S, Barnes VA et al. A genome-wide methylation study on obesity: differential variability and differential methylation. Epigenetics 8(5), 522-533 (2013).

21. Dick KJ, Nelson CP, Tsaprouni L et al. DNA methylation and body-mass index: a genome-wide analysis. Lancet 383(9933), 1990-1998 (2014).

22. Hidalgo B, Irvin MR, Sha J et al. Epigenome-wide association study of fasting measures of glucose, insulin, and HOMA-IR in the Genetics of Lipid Lowering Drugs and Diet Network Study. Diabetes 63(2), 801-807 (2014).

-• Reports the contradictious association between polymorphism rs9982016 and ABCG1 methylation at cg06500161 next to the association between ABCG1 methylation and Type 2 diabetes independently of BMI.

23. Chambers JC, Loh M, Lehne B et al. Epigenome-wide association of DNA methylation markers in peripheral blood from Indian Asians and Europeans with incident Type 2 diabetes: a nested case-control study. Lancet Diabetes Endocrinol. 3(7), 526-534 (2015).

- The first large-scale blood-based study $(n=25,372)$ showing an epigenome-wide association between SREBF1 and ABCG1 methylation and Type 2 diabetes. Moreover, a risk score to predict future diabetes risk was established.

24. Demerath EW, Guan W, Grove ML et al. Epigenome-wide association study (EWAS) of BMI, BMI change and waist circumference in African American adults identifies multiple replicated loci. Hum. Mol. Genet. 24(15), 4464-4479 (2015).

25. Kulkarni H, Kos MZ, Neary J et al. Novel epigenetic determinants of Type 2 diabetes in Mexican-American families. Hum. Mol. Genet. 24(18), 5330-5344 (2015).

26. Dayeh T, Tuomi T, Almgren P et al. DNA methylation of loci within ABCG1 and PHOSPHO1 in blood DNA is associated with future Type 2 diabetes risk. Epigenetics 11(7), 482-488 (2016).

- Similar to us, authors tried to replicate blood-based methylation marker in metabolically active tissue, whereby only $A B C G 1$ methylation in adipose tissue showed a promising association.

27. Wahl S, Drong A, Lehne B et al. Epigenome-wide association study of body mass index, and the adverse outcomes of adiposity. Nature 541(7635), 81-86 (2016). 
-• Reports the largest meta-analysis on DNA methylation in blood and tries to explain cause or consequence of methylation and obesity or Type 2 diabetes by statistical models and polymorphisms.

28. Walaszczyk E, Luijten M, Spijkerman AMW et al. DNA methylation markers associated with Type 2 diabetes, fasting glucose and HbA1c levels: a systematic review and replication in a case-control sample of the Lifelines study. Diabetologia 61(2), 354-368 (2018).

29. Pfeiffer L, Wahl S, Pilling LC et al. DNA methylation of lipid-related genes affects blood lipid levels. Circ. Cardiovasc. Genet. 8(2), 334-342 (2015).

30. Kriebel J, Herder C, Rathmann W et al. Association between DNA methylation in whole blood and measures of glucose metabolism: KORA F4 Study. PLoS ONE 11(3), e0152314 (2016).

31. Michels KB, Binder AM, Dedeurwaerder S et al. Recommendations for the design and analysis of epigenome-wide association studies. Nat. Methods 10(10), 949-955 (2013).

32. Dekkers KF, Van Iterson MFN, Slieker RC, et al. Blood lipids influence DNAmethylation in circulating cells. Genome Biol.17(1), 138 (2016).

33. Braun KVE, Dhana K, De Vries PS, et al. Epigenome-wide association study (EWAS) on lipids: the Rotterdam Study. Clin. Epigenetics9, 15 (2017).

34. Hedman AK, Mendelson MM, Marioni RE, et al. Epigenetic patterns in blood associated with lipid traits predict incident coronary heart disease events and are enriched for results from genome-wide association studies. Circ. Cardiovasc. Genet.10(1), (2017).

35. Sayols-Baixeras S, Subirana I, Lluis-Ganella C, et al. Identification and validation of seven new locishowing differential DNA methylation related to serum lipid profile: anepigenome-wide approach. The REGICOR study. Hum. Mol. Genet. 25(20), 4556-4565 (2016).

36. Truong V, Huang S, Dennis J, et al. Blood triglyceride levels are associated with DNA methylation at the serine metabolism gene PHGDH. Sci. Rep.7(1), 11207 (2017).

37. Dayeh T, Tuomi T, Almgren P, et al. DNA methylation of loci within ABCG1 and PHOSPHO1 inblood DNA is associated with future Type 2 diabetes risk. Epigenetics11(7), 482-488 (2016).

38. Akinyemiju T, Do AN, Patki A, et al. Epigenome-wide association study of metabolic syndrome in African-American adults. Clin. Epigenetics 10, 49 (2018).

39. Campanella G, Gunter MJ, Polidoro S, et al. Epigenome-wide association study of adiposity and future risk of obesity-related diseases. Int. J. Obes. (Lond.)42(12), 2022-2035 (2018).

40. Chen Y, Lemire M, Choufani S et al. Discovery of cross-reactive probes and polymorphic CpGs in the Illumina Infinium HumanMethylation 450 microarray. Epigenetics 8(2), 203-209 (2013).

-. Emphasizes the pitfalls of DNA methylation measurement by high-throughput techniques, in this case microarrays, and the urgent need for site-specific validation.

41. Rando OJ, Simmons RA. I'm eating for two: parental dietary effects on offspring metabolism. Cell 161(1), 93-105 (2015).

42. Davegårdh C, García-Calzón S, Bacos K, Ling C. DNA methylation in the pathogenesis of Type 2 diabetes in humans. Mol. Metab. 14 12-25 (2018).

43. Kennedy MA, Barrera GC, Nakamura K et al. ABCG1 has a critical role in mediating cholesterol efflux to HDL and preventing cellular lipid accumulation. Cell Metab. 1(2), 121-131 (2005).

44. Frisdal E, Le Lay $\mathrm{S}$, Hooton $\mathrm{H}$ et al. Adipocyte ATP-binding cassette G1 promotes triglyceride storage, fat mass growth, and human obesity. Diabetes 64(3), 840-855 (2015).

45. Buchmann J, Meyer C, Neschen S et al. Ablation of the cholesterol transporter adenosine triphosphate-binding cassette transporter G1 reduces adipose cell size and protects against diet-induced obesity. Endocrinology 148(4), 1561-1573 (2007).

- Shows the importance of $A B C G 1$ for regulating energy homeostasis using Abcg1-deficient mice.

46. Shao W, Espenshade PJ. Expanding roles for SREBP in metabolism. Cell Metab. 16(4), 414-419 (2012).

- Describes the importance of hepatic SREBF1 in Type 2 diabetes manifestation.

47. Oberkofler H, Fukushima N, Esterbauer H, Krempler F, Patsch W. Sterol regulatory element binding proteins: relationship of adipose tissue gene expression with obesity in humans. Biochim. Biophys. Acta. 1575(1-3), 75-81 (2002).

48. Chadwick LH, Sawa A, Yang IV et al. New insights and updated guidelines for epigenome-wide association studies. Neuroepigenetics 1, 14-19 (2015)

49. Reinius LE, Acevedo N, Joerink M et al. Differential DNA methylation in purified human blood cells: implications for cell lineage and studies on disease susceptibility. PLoS ONE 7(7), e41361 (2012).

50. Houseman E, Accomando WP, Koestler DC et al. DNA methylation arrays as surrogate measures of cell mixture distribution. $B M C$ Bioinformatics 13(1), 86 (2012).

51. Lin X, Song K, Lim N et al. Risk prediction of prevalent diabetes in a Swiss population using a weighted genetic score - the CoLaus Study. Diabetologia 52(4), 600-608 (2009).

52. Yarwood A, Han B, Raychaudhuri S et al. A weighted genetic risk score using all known susceptibility variants to estimate rheumatoid arthritis risk. Ann. Rheum. Dis. 74(1), 170-176 (2015). 
53. De Jager PL, Chibnik LB, Cui J et al. Integration of genetic risk factors into a clinical algorithm for multiple sclerosis susceptibility: a weighted genetic risk score. Lancet Neurol. 8(12), 1111-1119 (2009).

54. Ahmad AS, Vasiljević N, Carter P et al. A novel DNA methylation score accurately predicts death from prostate cancer in men with low to intermediate clinical risk factors. Oncotarget (2016). http://www.oncotarget.com/fulltext/12377 
